\title{
Photocatalytic activity of silica and silica-silver nanocolloids based on photo-induced formation of reactive oxygen species
}

\author{
G. Romolini ${ }^{1,2} \cdot$ M. Gambucci $^{1}$ - D. Ricciarelli ${ }^{1}$ - L. Tarpani ${ }^{1}$ - G. Zampini ${ }^{1} \cdot$ L. Latterini $^{1}$ (1)
}

Received: 22 July 2021 / Accepted: 8 August 2021 / Published online: 27 August 2021

(c) The Author(s) 2021

\begin{abstract}
Semiconductor nanomaterials are often proposed as photocatalysts for wastewater treatment; silica nanomaterials are still largely unexploited because their photocatalytic performances need improvements, especially under visible light. The present study is a proof-of-concept that amorphous silica colloids once submitted to the proper surface modifications change into an efficient photocatalyst even under low-energy illumination source. For this reason, silica-based colloidal nanomaterials, such as bare $\left(\mathrm{SiO}_{2} \mathrm{NPs}\right)$, aminated $\left(\mathrm{NH}_{2}-\mathrm{SiO}_{2} \mathrm{NPs}\right)$, and $\mathrm{Ag} \mathrm{NPs}$-decorated $\left(\mathrm{Ag}-\mathrm{SiO}_{2} \mathrm{NPs}\right)$ silica, are tested as photocatalysts for the degradation of 9-anthracenecarboxylic acid (9ACA), taken as a model aromatic compound. Interestingly, upon irradiation at $313 \mathrm{~nm}, \mathrm{NH}_{2}-\mathrm{SiO}_{2} \mathrm{NPs}$ induce 9ACA degradation, and the effect is even improved when $\mathrm{Ag}-\mathrm{SiO}_{2} \mathrm{NPs}_{\text {are }}$ used. On the other hand, irradiation at $405 \mathrm{~nm}$ activates the plasmon of $\mathrm{Ag}-\mathrm{SiO}_{2} \mathrm{NPs}$ photocatalyst, providing a faster and more efficient photodegradation. The photodegradation experiments are also performed under white light illumination, employing a lowintensity fluorescent lamp, confirming satisfying efficiencies. The catalytic effect of $\mathrm{SiO}_{2}$-based nanoparticles is thought to originate from photo-excitable surface defects and Ag NP plasmons since the catalytic degradation takes place only when the 9ACA is adsorbed on the surface. In addition, the involvement of reactive oxygen species was demonstrated through a scavenger use, obtaining a yield of $17 \%$. In conclusion, this work shows the applicability of silica-based nanoparticles as photocatalysts through the involvement of silica surface defects, confirming that the silica colloids can act as photocatalysts under irradiation with monochromatic and white light.
\end{abstract}

\section{Graphic abstract}

Silica and Ag-decorated silica colloids photosensitize the formation of Reactive Oxygen Species with 17\% efficiencies. ROS are able to oxidase aromatic pollutants chemi-adsorbed on the surface of the colloids. Silica-silver nanocomposites present a photocatalytic activity useful to degrade aromatic compounds.

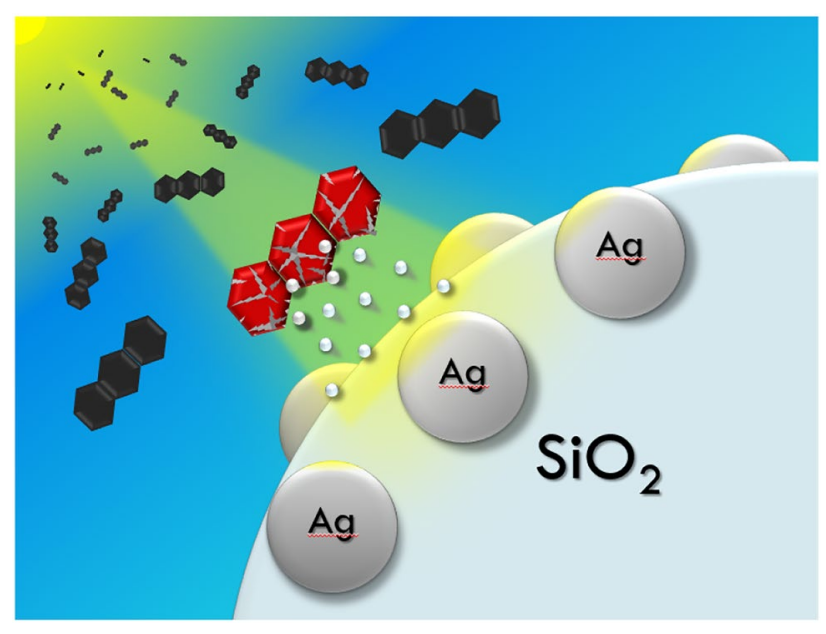

Extended author information available on the last page of the article 
Keywords Photocatalysis $\cdot$ Silica-silver nanocomposites $\cdot 9$-anthracenecarboxylic acid $\cdot$ Photodegradation $\cdot$ Reactive oxygen species

\section{Introduction}

Persistent organic pollutants are an increasing urgency that alter the composition of aquatic environments [1-3]. In addition to the well-known polycyclic aromatic hydrocarbons $[4,5]$ other contaminants, derived from pharmaceuticals, health-care products, drugs and pesticides [6-8], are increasingly being detected in watercourses and their presence can severely impact on the ecosystem.

This necessity fosters the development of sustainable methodologies to remove these pollutants.

One promising strategy, that has attracted considerable interest, is based on photocatalysts [9-11], i.e. materials able to induce the degradation of a wide range of organic pollutants once triggered by radiation [12-14].

Several semiconductors $\left(\mathrm{TiO}_{2}, \mathrm{SnO}_{2}, \mathrm{ZrO}_{2}, \mathrm{Fe}_{2} \mathrm{O}_{3}, \mathrm{ZnO}\right.$, $\mathrm{ZnS}, \mathrm{CdS}$ ) nanoparticles have been studied as photocatalysts $[15,16]$ and, among them, $\mathrm{TiO}_{2}$ and $\mathrm{ZnO}$ nanomaterials have been the most investigated due to their good photocatalytic performances [17-19].

Silica nanomaterials $\left(\mathrm{SiO}_{2} \mathrm{NPs}\right)$ represent an appealing alternative because they guarantee lower environmental impact and an easily processable surface for post-synthesis functionalization [20-22]. However, the use of silica-based materials as photocatalysts has been strongly limited by their electronic properties. Indeed, crystalline silica has a very wide bandgap (approximately $9 \mathrm{eV}$ [23]) which makes it transparent to UVB, UVA and visible radiations, making difficult their photoexcitation in natural irradiation conditions.

However, the presence of structural defects turns silica photoactivable with UV radiation; several defects have been studied and characterized for amorphous silica nanoparticles $[24,25]$, such as non-bridging oxygen hole centers and neutral deficient oxygen centers. These defects possess optical absorption covering the UVB and UVA regions, which are able to enhance catalytic properties of $\mathrm{SiO}_{2} \mathrm{NPs}$ under UV irradiation [26, 27].

However, to make the photocatalytic systems even more appealing and efficient, there is the need to extend the catalyst activation in the visible range, to take advantage of the sunlight radiation which is mainly composed of visible photons $(40-50 \%)$ [28].

One feasible strategy is given by the combination of semiconductor nanomaterials with metal nanoparticles, whose surface plasmon resonance (SPR) absorption can be shifted from UV to IR range [29]. The plasmon excitation leads to an enhanced photocatalytic ability of the nanocatalyst, mainly for two reasons: firstly, the hot-carrier injection from the metal to the semiconductor can enhance the abundance of charge carriers within the nanomaterial, thus increasing the number of catalytic active sites [30,31]. Secondly, the photogenerated hot electrons can be collected by the species adsorbed on the semiconductor surface, such as dyes, water or $\mathrm{O}_{2}$, generating highly reactive radicals [32].

For instance, $\mathrm{He}$ and coworkers [33] demonstrated that the hot-carrier injection was possible with nickel- $\mathrm{TiO}_{2}$ heterostructure. They found that both the hot electrons and hot holes, generated by the plasmon excitation of nickel, migrated from $\mathrm{Ni}$ to $\mathrm{TiO}_{2}$. The transferred hot electrons tend to occupy the oxygen vacancies on $\mathrm{TiO}_{2}$, while the hot holes are located on the surface oxygens of the semiconductor. Moreover, they pointed out that hot electrons dominantly contributed to the degradation of methylene blue. Saravanan and coworkers [34] revealed that the conjugation of silver to $\mathrm{TiO}_{2}$ induce structural/electronic perturbation that alters the bandgap of the semiconductor and prevents the charge-carrier recombination. These findings result in good photocatalytic efficiency under visible light for the degradation of methyl orange and for the photoinduced hydrogen production through water splitting. Raji et al. [35] reported the successful photodegradation of sulforhodamine $\mathrm{B}$ under sunlight with $\mathrm{ZnO} / \mathrm{Ag}$ nanorods as catalyst; in particular, the increase of adsorbed Ag led to an enhanced photocatalytic activity, which was assigned to the improved production of reactive oxygen species. Indeed, the formation of reactive oxygen species (ROS) has been demonstrated to be relevant in photocatalytic degradation of organic pollutants in aqueous media [36]. Therefore, the development of efficient and sustainable photocatalysts can be based in growth of efficient ROS photosensitizers [36]; their photosensitization efficiency is an important parameter to be quantitatively determined.

Mesoporous silica has been widely used as a support or templating material [37-39], but its photocatalytic performances have been barely investigated [40, 41].

Here, we explore the photocatalytic properties of silica colloids and the possibility to exploit the system under visible irradiation upon conjugation of silver nanoparticles; we determined the efficiency of various silica-based nanomaterials in the degradation of 9-anthracenecarboxylic acid (9ACA), which is used as model aromatic compound for persistent organic pollutants. Bare silica nanoparticles have been prepared by a sol-gel method and then functionalized with a post-synthesis process with amino groups to investigate the role of surface chemistry on the aromatic compound photodegradation. Finally, after the conjugation of silver nanoparticles to silica colloids, the photocatalytic performance under visible light has been measured. Moreover, to 
achieve insight on the potential use of the proposed systems, the irradiation with a common white light source (WL) has been performed as well. To disclose the photocatalytic mechanism, the analysis and quantification of ROS generation have been carried out.

\section{Experimental section}

\subsection{Materials}

9-Anthracenecarboxylic acid (9ACA, 99\%), tetraethylorthosilicate (TEOS, 98\%), (3-aminopropyl) triethoxysilane (APTES, $\geq 98 \%$ ), cetyltrimethylammonium tosilate (CTATos, 95\%), triethanolamine $\left(\mathrm{TEAH}_{3},>98 \%\right)$, 1,3-diphenylisobenzofuran (DPBF, 97\%), rose bengal $(\mathrm{RB}, \geq 95 \%)$, silver nitrate $\left(\mathrm{ANNO}_{3}, \geq 99.9 \%\right)$, sodium borohydride $\left(\mathrm{NaBH}_{4}, \geq 99 \%\right)$ and 3-mercaptopropionic acid (MPA, $\geq 99 \%$ ) were all purchased from Sigma-Aldrich and used as received. Nanopure water $(\leq 15.0 \mathrm{M} \Omega)$ from a Millipore Milli-Q gradient system and ethanol (96\%) from Sigma-Aldrich were used as solvents.

\subsection{Silica nanoparticles synthesis}

Silica nanoparticles ( $\left.\mathrm{SiO}_{2} \mathrm{NPs}\right)$ are synthesized with a procedure described elsewhere, using the sol-gel method in the presence of a surfactant as templating agent [42].

Briefly, in a $100 \mathrm{~mL}$ flask equipped with a magnetic bar, $0.95 \mathrm{~g}$ of cetyltrimethylammonium tosilate (CTATos), 120 $\mu \mathrm{L}$ of triethanolamine $\left(\mathrm{TEAH}_{3}\right)$ and $50 \mathrm{~mL}$ of water are added and stirred at $80{ }^{\circ} \mathrm{C}$. After $1 \mathrm{~h}, 7.69 \mathrm{~mL}$ of tetraethylorthosilicate (TEOS) are quickly added, and the mixture is left under stirring for 2 additional hours at $80{ }^{\circ} \mathrm{C}$. At the end of the reaction, the mixture is cooled in a cold water bath for about $10 \mathrm{~min}$. The nanoparticles are recovered by centrifugation at $3000 \mathrm{~g}$ for $30 \mathrm{~min}$ and then washed several times with ethanol. Finally, the incorporated (or pore occluding) organic surfactants are removed through calcination at $550{ }^{\circ} \mathrm{C}$ for $6 \mathrm{~h}$.

Post-synthesis treatment to graft amino groups on the surface of $\mathrm{SiO}_{2} \mathrm{NPs}$ is performed in ethanol with a procedure described elsewhere [43], using $\mathrm{SiO}_{2} \mathrm{NPs}$ in concentration of $2.0 \mathrm{mg} \mathrm{mL}^{-1}$ and $8 \mu \mathrm{L}$ of APTES per $\mathrm{mL}$ of ethanol.

\subsection{Silver nanoparticles synthesis}

$25.0 \mathrm{~mL}$ of a $2.0 \mathrm{mM}$ aqueous solution of sodium borohydride $\left(\mathrm{NaBH}_{4}\right)$ are stirred in an ice bath. Then, $0.8 \mathrm{~mL}$ of a $1.2 \mathrm{mM}$ solution of silver nitrate $\left(\mathrm{AgNO}_{3}\right)$ are added dropwise. After $10 \mathrm{~min}, 5.0 \mathrm{~mL}$ of an aqueous solution $0.050 \mathrm{mM}$ of 3-mercaptopropionic acid (MPA) are added to $5.0 \mathrm{~mL}$ of the yellow colloidal solution and stirred for 5 additional minutes. The as-synthesized silver nanoparticles (Ag NPs) are stored in dark at room temperature.

\subsection{Adsorption procedure of silver nanoparticles onto silica nanoparticles surface}

$10 \mathrm{~mL}$ of Ag NPs colloidal solution are added in a $25 \mathrm{~mL}$ flask and put it in an ultrasound bath; then $10 \mathrm{mg}$ of $\mathrm{NH}_{2}-\mathrm{SiO}_{2} \mathrm{NPs}$ are added very slowly. After the formation of a fine suspension, the system is transferred to a magnetic stirrer and kept under vigorous stirring for $2 \mathrm{~h}$. The silversilica nanoparticles $\left(\mathrm{Ag}-\mathrm{SiO}_{2} \mathrm{NPs}\right)$ are collected through centrifugations steps, to separate the $\mathrm{Ag}-\mathrm{SiO}_{2} \mathrm{NPs}$ from the non-adsorbed Ag NPs; the centrifugation is repeated until the surnatant contained silver nanoparticles. Finally, the obtained pellet is washed three times with ethanol and then dried under a gentle flux of nitrogen. The amount of adsorbed Ag NPs is derived by the analysis of the extinction spectrum recorded before (starting colloidal solution) and after (supernatants) the anchoring procedure.

\subsection{Photocatalytic degradation of 9-anthracenecarboxylic acid by various silica nanocomposites}

Photodegradation analysis of 9-anthracenecarboxylic acid (9ACA) is carried out by varying either the concentration of silica nanocomposites or the irradiation wavelength, while every other experimental setup is kept constant. The time-dependent decrease of 9ACA concentration under irradiation is followed through fluorimetric assays; indeed, the photocatalytic pathway generates non-fluorescent products [44] which makes it easy to follow the degradation of 9ACA through the decrease of the relative fluorescence emission.

In a typical experiment, $200 \mu \mathrm{L}$ of 9ACA solution $\left(1.1 \times 10^{-4} \mathrm{M}\right.$ in water) are placed in a cuvette (with $1 \mathrm{~cm}$ optical path length) and diluted with $2 \mathrm{~mL}$ of an aqueous suspension of silica (each nanocomposite is in concentration of $1.0 \mathrm{mg} \mathrm{mL}^{-1}$ ). Then, the overall system is irradiated with a mercury lamp or a WL under continuous stirring, while fluorescence spectra are acquired at specific time intervals, up to $120 \mathrm{~min}$ of irradiation.

The photocatalytic efficiency of the nanocomposites and the kinetic profiles of the photodegradation of 9ACA, are calculated similarly to Selvaggi et al. [45]. All the reported data are mean values of three independent experiments.

Similarly, a control experiment with AgNPs was performed. The AgNPs were diluted to obtain an extinction value between 0.2 and 0.25 at $400 \mathrm{~nm}$. To this $200 \mu \mathrm{L}$ of 9ACA solution $\left(1.1 \times 10^{-4} \mathrm{M}\right.$ in water $)$ were added before irradiation. 


\subsection{Quantitative analysis of the photosensitized reactive oxygen species}

The photo-induced quantum efficiency of ROS formation is determined through a widely used ROS trap, 1,3-diphenylisobenzofuran (DPBF). The data are analyzed with an already reported model [43] (see also Supporting Information). Since a white light source is adopted, instead of monochromatic irradiation, the absorbance values of the standard and the sample under investigation are integrated into the whole UV-VIS range [46].

The used sample concentration $\left(\mathrm{Ag}-\mathrm{SiO}_{2} \mathrm{NPs}\right)$ is $0.5 \mathrm{mg} \mathrm{mL}^{-1}$ in all the experiments and DPBF concentration is fixed to ca. $50 \mu \mathrm{M}$. The experiment is performed in ethanol.

\subsection{Instrumentation}

\subsubsection{Optical characterization}

Diffuse reflectance UV-VIS spectra of powder samples are recorded with a Varian Cary 4000 spectrophotometer equipped with a $150-\mathrm{mm}$ integration sphere; a barium sulfate tablet is used as reference. The absorption and extinction spectra of solutions and nanoparticle suspensions are collected through a Cary 8454 UV-VIS Diode Array spectrophotometer. The photocatalytic degradation analysis is performed by acquiring the fluorescence emission spectra of 9ACA $\left(\lambda_{\text {exc }}=363 \mathrm{~nm}\right)$ on a Fluorolog-2 (Spex, F112AI) fluorimeter.

\subsubsection{Morphological and structural characterization}

A Philips 208 transmission electron microscope (TEM) with $80 \mathrm{kV}$ of beam acceleration is used to acquire the nanoparticle images by depositing one drop of each suspension on a 300 square mesh Formvar-coated copper grid. The relative histograms are derived from ImageJ (Rasband, W.S., ImageJ, U. S. National Institutes of Health, Bethesda, Maryland, USA, http://imagej.nih.gov/ij/, 1997-2016), and the mean diameters are calculated from the best Gaussian fit of the experimental data.

Energy dispersive X-ray microanalysis (EDX) spectroscopy supported by a field emission scanning electron microscope (FE-SEM) (FEG LEO 1525) is used to analyze both the elemental composition and the morphology of $\mathrm{Ag}-\mathrm{SiO}_{2}$ sample.

\subsubsection{Radiation sources for photocatalysis experiments}

The irradiation experiments are performed using two different lamps. The first one is a mercury-vapor lamp operating at high pressure $(500 \mathrm{~W})$ equipped with bandpass filters at $313 \pm 15 \mathrm{~nm}$ (for the excitation of silica defects) or $405 \pm 15 \mathrm{~nm}$ (for the photoactivation of Ag NPs plasmon); the second source is a white-light fluorescent lamp (WL) for indoor lighting (Beghelli FLUO Tli8 TRIMAX 18 W). From the manufacturer's specifications, it is estimated a WL irradiance of about $33 \mathrm{~W} / \mathrm{m}^{2}$. The irradiance spectra of both lamps are reported in the supporting info (Figs. S13 and S14).

\section{Results and discussion}

\subsection{Morphological and optical characterization of silica nanocomposites}

Following the procedures described in the experimental section, silica nanoparticles are prepared. The hydrolysis of tetraethylorthosilicate (TEOS) at $\mathrm{pH} \sim 7$ and using cetyltrimethylammonium tosilate (CTATos) as templating agent $[42,47]$, results in silica nanoparticles $\left(\mathrm{SiO}_{2} \mathrm{NPs}\right)$ with mesoporous channel-like structure. The tight control of the synthesis conditions enables to obtain $\mathrm{SiO}_{2} \mathrm{NPs}$ with uniform shape and dimensions presenting an average diameter of about $120 \pm 9.7 \mathrm{~nm}$ (Figs. 1a and S1). The mesoporous structure of silica increases the exposed surface area, as documented for similar materials, where the formation of channel-like structure on silica, more than doubled the material surface area [48] and hence its contact area.

The outer surface of $\mathrm{SiO}_{2}$ NPs is then modified by grafting 3-aminopropyltriethoxysilane (APTES), to obtain amino-functionalized nanoparticles, namely $\mathrm{NH}_{2}-\mathrm{SiO}_{2}$ NPs. The successful surface functionalization with APTES is demonstrated by FTIR spectra of $\mathrm{NH}_{2}-\mathrm{SiO}_{2} \mathrm{NPs}$ and $\mathrm{SiO}_{2}$ NPs (Fig. S1); the presence of additional peaks in the spectrum of $\mathrm{NH}_{2}-\mathrm{SiO}_{2} \mathrm{NPs}$ at 2850 and $2920 \mathrm{~cm}^{-1}$ assigned to the asymmetric and symmetric $\mathrm{CH}_{2}$ stretching, respectively, are due to propyl chain of grafted APTES, while the weak peaks at 1555 and $1537 \mathrm{~cm}^{-1}$ are due to $\mathrm{N}-\mathrm{H}$ stretching. The peak at $1740 \mathrm{~cm}^{-1}$ could be tentatively assigned to the $\mathrm{CO}_{2}$ adsorbed on the amino-functionalized silica surface.

The presence of amino groups modifies the silica surface charge distribution, as indicated by zeta potential values measured for similar materials $(-62 \mathrm{mV}$ for bare mesoporous silica [47] and $+12 \mathrm{mV}$ after the amino functionalization [48]), but also affects the optical properties of silica powders, as evidenced by the reflectance spectra of the samples (Fig. 2a), likely altering the electronic structure of the silica surface. The grafting procedure of amino groups does not affect the morphology and the dimension of the particles, as indicated by the analysis of TEM images (see Figs. S2-S4 in supporting information for the size distribution analysis).

Previously prepared silver nanoparticles have been anchored on the silica surface, taking advantage of the 


\section{(a)}

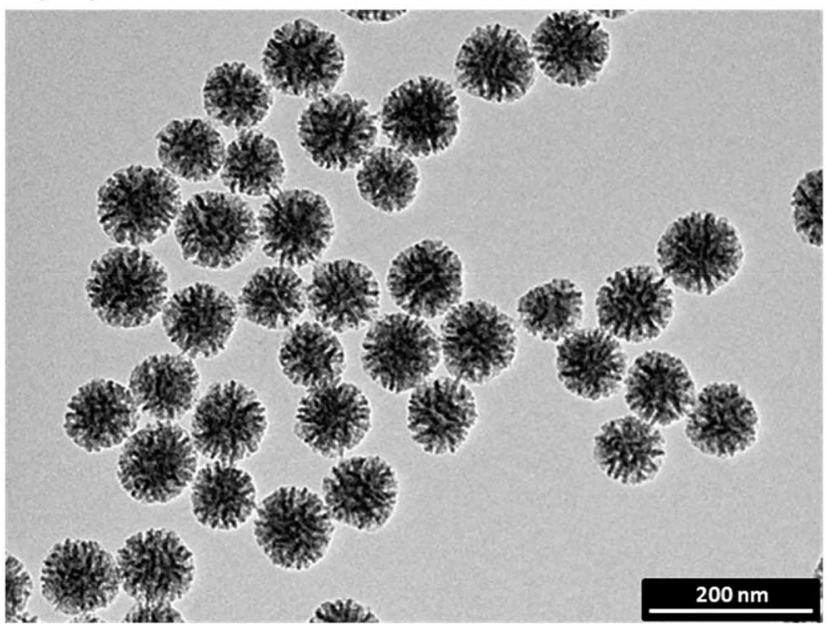

(c)

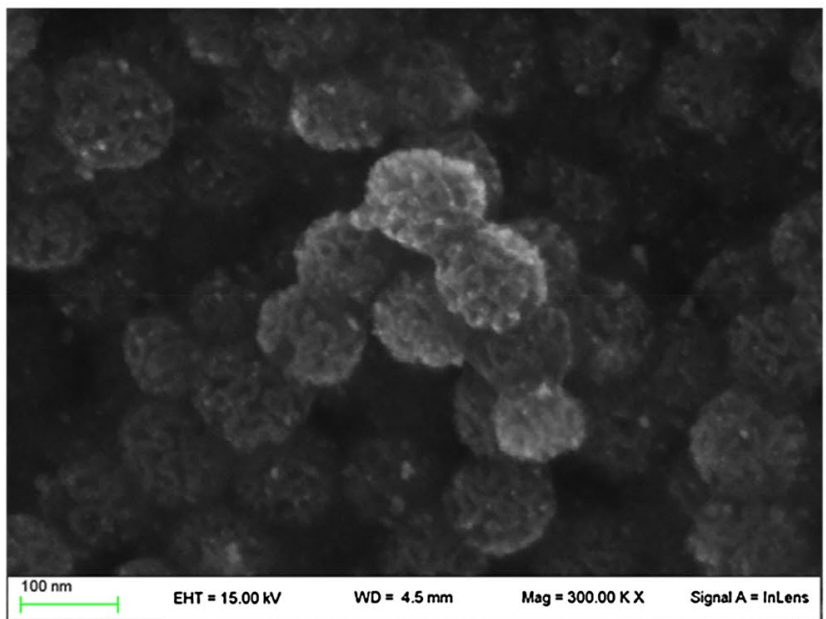

Fig. 1 TEM images of: a $\mathrm{SiO}_{2}$ NPs (scale bar $200 \mathrm{~nm}$ ) and b $\mathrm{Ag}$ $\mathrm{SiO}_{2} \mathrm{NPs}$ (scale bar $100 \mathrm{~nm}$ ); inset magnification of a single $\mathrm{Ag}-\mathrm{SiO}_{2}$ NP (the red arrows indicate the AgNPs). c SEM image and d relative

amino-functionalized surface. Silver colloids (Ag NPs), with a mean diameter of $10 \pm 3 \mathrm{~nm}$ (Fig. S4) have been formerly synthesized through a selective wet reduction (see experimental section) using 3-mercaptopropionic acid (MPA) as a stabilizer. Ag NPs are chemi-adsorbed on the surface of $\mathrm{NH}_{2}-\mathrm{SiO}_{2} \mathrm{NPs}$ by contacting the colloidal suspensions. The substantial dissociation of MPA ( $\mathrm{pKa}=4.3$ for the carboxylic group), at neutral $\mathrm{pH}$ conditions, drives the establishment of electrostatic interactions with the amino groups on the silica surface, forming silica-silver nanocomposites ( $\left.\mathrm{Ag}-\mathrm{SiO}_{2} \mathrm{NPs}\right)$. The spectrophotometric analysis of the silver suspension, before and after the contact with the aminated silica, enables to estimate an anchoring efficiency equal to $85 \%$ (Fig. S5). Therefore, (b)

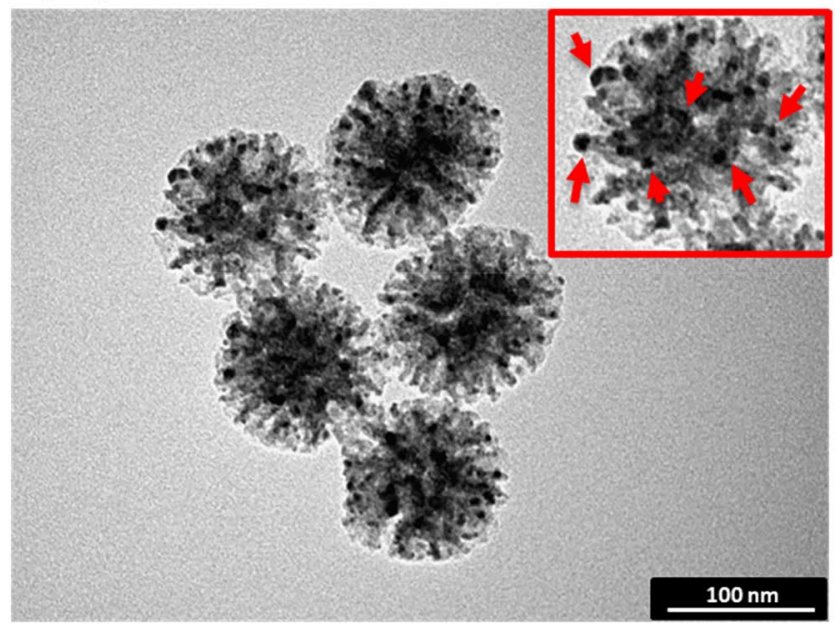

(d)

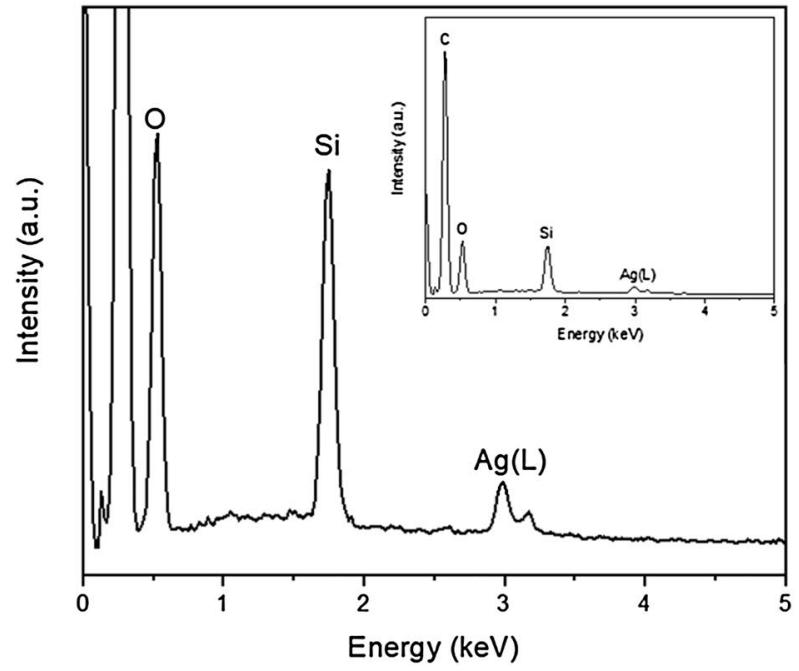

EDX spectrum of $\mathrm{Ag}-\mathrm{SiO}_{2}$ NPs (the complete EDX spectrum is displayed as an inset)

the estimated amount of adsorbed silver is $1.7 \mu g_{A g} / m_{S i O_{2}}$. Moreover, it is possible to confirm the adsorption of $\mathrm{Ag}$ NPs on silica with TEM and SEM imaging, as shown in Fig. 1b, c. The elemental composition through EDX analysis (Fig. 1d) confirms the presence of silver throughout the analyzed sample.

The reflectance spectra of the silica samples in powder form (Fig. 2a) display the lowest energy bands located in the 200-350 $\mathrm{nm}$ range [49], likely due to the presence of surface defects [45].

The presence of Ag NPs on the silica surface significantly broadens the visible response of $\mathrm{Ag}-\mathrm{SiO}_{2} \mathrm{NPs}$ colloids (Fig. 2a, b) for the attendance of surface plasmon resonance band, centered at $405 \mathrm{~nm}$. 
(a)

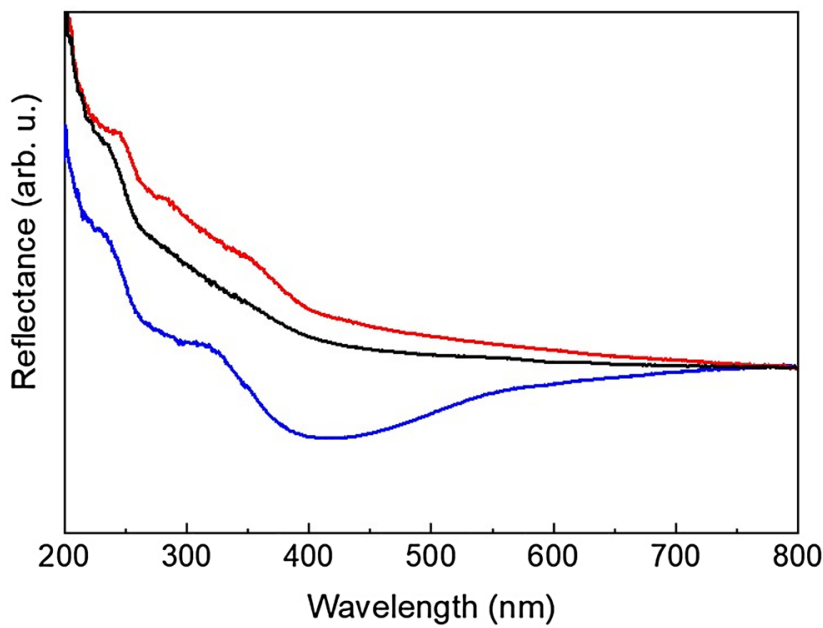

(b)

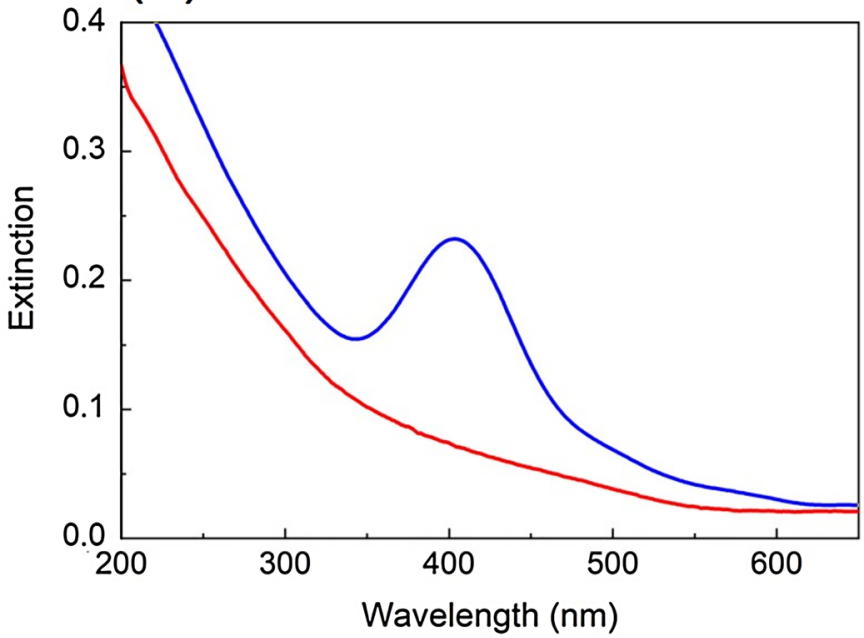

Fig. 2 a Reflectance spectra of $\mathrm{SiO}_{2} \mathrm{NPs}$ (black line) $\mathrm{NH}_{2}-\mathrm{SiO}_{2} \mathrm{NPs}$ (red line) and $\mathrm{Ag}-\mathrm{SiO}_{2} \mathrm{NPs}$ (blue line) powders. b Extinction spectra of aqueous $\mathrm{NH}_{2}-\mathrm{SiO}_{2} \mathrm{NPs}$ (red line) and $\mathrm{Ag}-\mathrm{SiO}_{2} \mathrm{NPs}$ (blue line) suspensions $(0.5 \mathrm{mg} / \mathrm{mL}$ )

The extinction spectrum of an aqueous suspension of $\mathrm{Ag}$ $\mathrm{SiO}_{2}$ NPs is monitored for $72 \mathrm{~h}$ (Fig. S5); the spectra display only a slight red-shift of the absorption maximum, from 405 to $407 \mathrm{~nm}$, thus indicating the substantial stability of the nanocomposite in suspension.

\subsection{Photocatalytic degradation of 9ACA through monochromatic light}

The effect of surface functionalization of the colloids has been explored by monitoring their photocatalytic activity

(a)

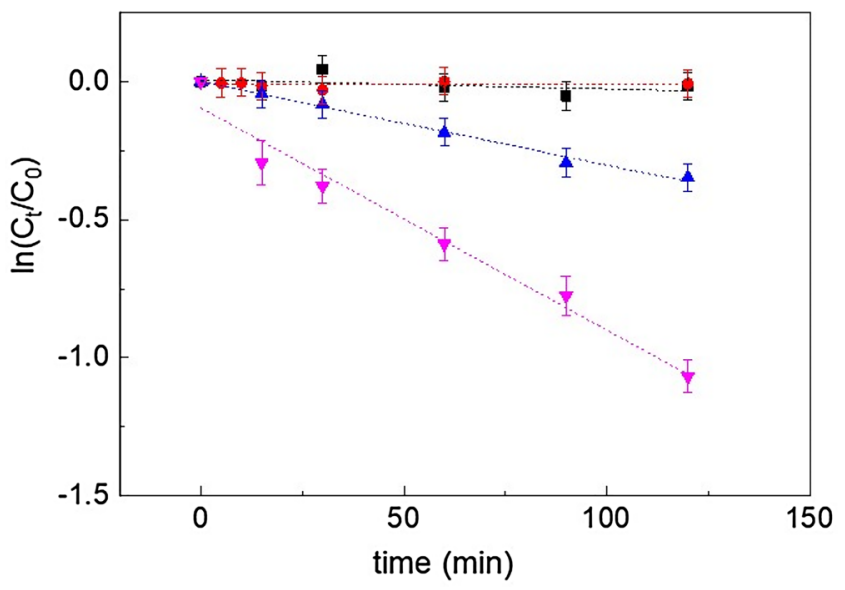

Fig. 3. 9ACA photodegradation kinetics performed in water, in absence of nanoparticles (Black box) and presence of $\mathrm{SiO}_{2} \mathrm{NPs}$ (Orange circle), $\mathrm{NH}_{2}-\mathrm{SiO}_{2} \mathrm{NPs}$ (Blue Triangle), and $\mathrm{Ag}-\mathrm{SiO}_{2}$ on the degradation of 9ACA, irradiating at $313 \mathrm{~nm}$ or $405 \mathrm{~nm}$, to selectively photo-activate the silica or silver, respectively. The 9ACA concentration is tracked by following the change of its fluorescence emission intensity at $409 \mathrm{~nm}$ at different irradiation times (Figs. 3 and S7). The reduction of the emission intensity is correlated to the decrease of 9ACA concentration since the photodegradation pathway generates non-fluorescent products [44]. As a control experiment, the same irradiation setup is used on the 9ACA solution without the colloids; the changes of 9ACA fluorescence intensity in the presence of silica

(b)

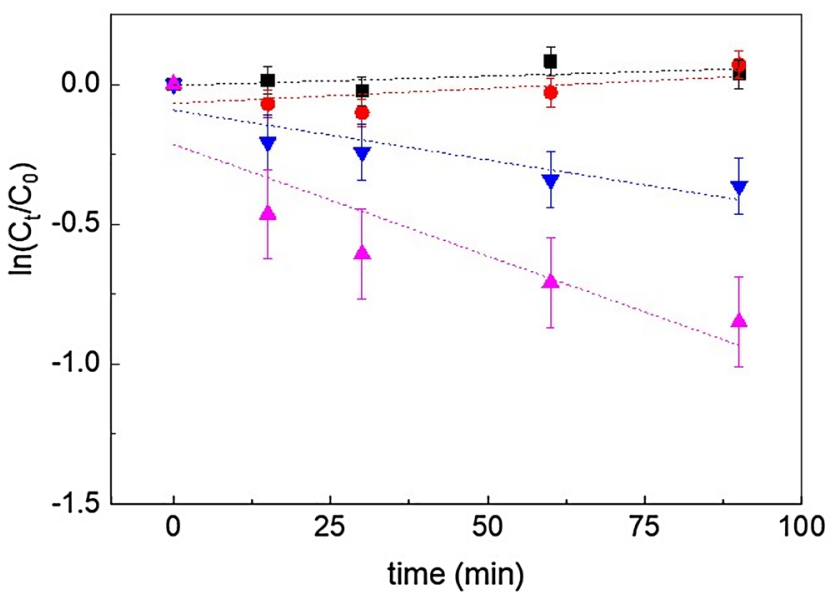

NPs (Purple Down Triangle) in concentration of $1.0 \mathrm{mg} \mathrm{mL}^{-1}$ a $\lambda_{\text {irr }}=313 \mathrm{~nm} ; \mathbf{b} \lambda_{\text {irr }}=405 \mathrm{~nm}$. The dotted lines are the linear fits of the data 
Table 1 Parameters for the photodegradation of 9ACA $\left(\lambda_{\text {irr }}=313 \mathrm{~nm}\right)$

\begin{tabular}{llll}
\hline Catalyst & $\begin{array}{l}\text { Photocatalytic } \\
\text { efficiency } \%^{\mathrm{a}}\end{array}$ & $\mathrm{k}\left(10^{-3} \mathrm{~min}^{-1}\right)$ & $\begin{array}{l}\text { Half-time } \\
\mathrm{t}_{1 / 2}(\mathrm{~min})\end{array}$ \\
\hline- & 0 & 0 & - \\
$\mathrm{SiO}_{2} \mathrm{NPs}$ & 0 & 0 & - \\
$\mathrm{NH}_{2}-\mathrm{SiO}_{2} \mathrm{NPs}$ & 29 & $3.0 \pm 0.1$ & $230 \pm 80$ \\
$\mathrm{Ag}_{-} \mathrm{SiO}_{2} \mathrm{NPs}$ & 66 & $8.1 \pm 0.9$ & $85 \pm 10$ \\
\hline
\end{tabular}

9ACA concentration $1.0 \times 10^{-5} \mathrm{M}$; catalyst concentration $1.0 \mathrm{mg} \mathrm{mL}^{-1}$

${ }^{a}$ Measured after 120 min of irradiation

colloids is, within the experimental errors, negligible when the samples are stored in the dark.

The time evolution data of 9ACA upon irradiation at $313 \mathrm{~nm}$, in the different experimental conditions, are collected in Fig. 3a; Table 1 summarizes the obtained results, in terms of process efficiency (in the used experimental conditions), photocatalytic rate constant and half-life $t_{1 / 2}$ of 9ACA during degradation. As a reference, the results in the absence of the catalyst are reported as well. The data evidence that 9ACA in aqueous solution (in the absence of any colloids) and even in the presence of $\mathrm{SiO}_{2} \mathrm{NPs}$, is stable and the irradiation at $313 \mathrm{~nm}$ does not produce any decrease of the anthracene concentration. Interestingly, when $\mathrm{NH}_{2}-\mathrm{SiO}_{2}$ NPs are used as a catalyst, a reduction of 9ACA concentration up to $30 \%$ is measured after two hours of irradiation. Using $\mathrm{Ag}-\mathrm{SiO}_{2} \mathrm{NPs}$ under $313 \mathrm{~nm}$ irradiation, a remarkable degradation of 9ACA is found; in two hours almost $70 \%$ of 9ACA is photodegraded (see Table 1), which is than more than double the values reached with $\mathrm{NH}_{2}-\mathrm{SiO}_{2} \mathrm{NPs}$.

In these experimental conditions, the $313 \mathrm{~nm}$ radiation is mainly absorbed by the silica core of the nanomaterials (see Fig. S8 for 9ACA absorption spectrum), making the photocatalysts active to induce the photodegradation of 9ACA.

On the bases of our results, we ascribe the silica catalytic performances to the activation of non-bridging oxygen hole centers $\left(\equiv \mathrm{Si}-\mathrm{O}^{*}\right)$ surface defects which show $\sim 305 \mathrm{~nm}(\sim 4 \mathrm{eV})$ absorption contribution [24, 25, 50], comparable to the absorption band reported in Fig. S5. The excitation of this kind of defect leads to a charge-transfer process from $\mathrm{Si}-\mathrm{O}$ bonding orbital to a non-bonding orbital of non-bridging oxygen [50]. This charge separated state has a typical duration of ca. 10-20 $\mu \mathrm{s}[15,36]$, which is long enough to favor the charge transfer to the surrounding molecules rather than the direct relaxation of this excited state.

However, to make the photodegradation process effective, it is essential that interactions between the catalyst surface and 9ACA occur, as demonstrated by our results. The absence of any photocatalytic activity in bare $\mathrm{SiO}_{2} \mathrm{NPs}$ can be related to the establishment of repulsive interactions between the particles and the anthracene derivative, since both species are negatively charged in water. Instead, the amino groups, grafted on the silica surface, are able to establish attractive interactions with 9ACA, thus allowing the organic compound to approach the surface of the semiconductor and enhancing the photocatalytic efficiency up to $30 \%$, as previously observed for the photodegradation of acridine orange in water [45].

The electron-acceptor character of the metal nanoparticles can account for the higher photocatalytic performance of $\mathrm{Ag}-\mathrm{SiO}_{2} \mathrm{NPs}$. Indeed, once the silica defects are photoactivated by $313 \mathrm{~nm}$ radiation and form charge separated species, the hot electrons can migrate towards the metal. This can extend even further the lifetime of silica separated charged species, thus increasing the probability of the interactions with 9ACA [30, 31].

When the colloids under investigation $\left(\mathrm{NH}_{2}-\mathrm{SiO}_{2} \mathrm{NPs}\right.$ and $\mathrm{Ag}-\mathrm{SiO}_{2} \mathrm{NPs}$ ) are irradiated at $405 \mathrm{~nm}$, degradation of 9ACA is observed (Fig. 3b), although to different extents; the photodegradation kinetic parameters, determined from fluorescence measurements, are summarized in Table 2. In the presence of $\mathrm{Ag}-\mathrm{SiO}_{2} \mathrm{NPs}, 60 \%$ photodegradation of 9ACA is achieved after two hours of irradiation (Table 2) with monochromatic light at $405 \mathrm{~nm}$, where it is essentially absorbed by metal nanostructures. Only a slight decrease of 9ACA concentration is observed in the presence of $\mathrm{NH}_{2}-\mathrm{SiO}_{2} \mathrm{NPs}$ (about $6 \%$ of the degradation observed with $\mathrm{Ag}-\mathrm{SiO}_{2} \mathrm{NPs}$ ) and no reduction is detected in the absence of particle.

This VIS-activated photocatalytic efficiency is undoubtedly related to the surface plasmon resonance (SPR) of Ag NPs [51]. The high extinction coefficient and the broad absorption of SPR allow the most effective and wider-wavelength activation of the nanocomposite.

The hot-carriers, generated by the plasmon excitation, can directly react with adsorbed molecules (that can be the anthracene substrates, $\mathrm{O}_{2}$ or water molecules), generating extremely reactive radical species able to degrade 9ACA. However, the involvement of the silica is supported by the lack of observing relevant changes in the 9ACA concentration when irradiated in the presence of simple Ag-colloids (Fig. S15).

Table 2 Parameters for the photodegradation of 9ACA $\left(\lambda_{\text {irr }}=405 \mathrm{~nm}\right)$

\begin{tabular}{|c|c|c|c|}
\hline Catalyst & $\begin{array}{l}\text { Photocatalytic } \\
\text { efficiency } \%^{a}\end{array}$ & $\mathrm{k}\left(10^{-3} \min ^{-1}\right)$ & $\begin{array}{l}\text { Half-time } \\
\mathrm{t}_{1 / 2}(\min )\end{array}$ \\
\hline- & 0 & 0 & - \\
\hline $\mathrm{NH}_{2}-\mathrm{SiO}_{2} \mathrm{NPs}$ & 3.5 & $0.5 \pm 0.1$ & $1390 \pm 280$ \\
\hline $\mathrm{Ag}-\mathrm{SiO}_{2} \mathrm{NPs}$ & 60 & $8.0 \pm 0.9$ & $90 \pm 10$ \\
\hline $\begin{array}{l}\text { 9ACA conce } \\
1.0 \mathrm{mg} \mathrm{mL}^{-1}\end{array}$ & $1.0 \times 10^{-5}$ & M; catalyst & concentration \\
\hline
\end{tabular}




\subsection{Photocatalytic degradation of 9ACA under white light irradiation}

To evaluate the relevance of these materials as photocatalysts, their photocatalytic performances are tested also under irradiation with a white light (WL) lamp, mimicking indoor illumination, which displays a higher emission component in the visible range. The results are reported in Table 3 and Fig. S8.

As expected, $\mathrm{SiO}_{2}$ NPs do not display any significant photocatalytic activity, whereas, the silver-silica nanocomposite is the most efficient catalyst, with a degradation rate constant doubled compared to $\mathrm{NH}_{2}-\mathrm{SiO}_{2} \mathrm{NPs}$. Indeed, $\mathrm{NH}_{2}-\mathrm{SiO}_{2} \mathrm{NPs}$ absorb only the UV portion of the lamp radiation, while the $\mathrm{Ag}-\mathrm{SiO}_{2} \mathrm{NPs}$ absorb also in the visible range, thus a larger percentage of the photons emitted by the WL are able to activate the metal-functionalized photocatalyst. The smaller efficiencies measured with WL are related to the lower photon flux of the used source compared to the monochromatic irradiation.

\subsection{Efficiencies of ROS production under white light irradiation}

It has already been reported that under the proper illumination the silica defects can be activated [26] and generate hydroxyl radical $\left(\mathrm{OH}^{\bullet}\right)$ or superoxide radical anion $\left(\mathrm{O}_{2}{ }^{\bullet-}\right)$ [45], which are extremely reactive against organic chemicals adsorbed on the surface of the catalyst.

Our photodegradation experiments indicate that the activation of both silica defects and silver SPRs can generate charge separated carriers, whose interaction with the surrounding water or oxygen molecules may bring to the production of reactive oxygen species (ROS). However, the quantum efficiency of ROS photosensitization has not been determined.

Therefore, the ability of $\mathrm{Ag}-\mathrm{SiO}_{2} \mathrm{NPs}$ to photo-induce the formation of ROS is evaluated using 1,3-diphenylisobenzofuran (DPBF, see experimental section) which reacts selectively with $\mathrm{O}_{2}{ }^{\bullet-}$ and ${ }^{1} \mathrm{O}_{2}$ resulting in colorless products [52].

Table 3 Parameters for the photodegradation of 9ACA under irradiation through white light

\begin{tabular}{|c|c|c|c|}
\hline Catalyst & $\begin{array}{l}\text { Photocatalytic } \\
\text { efficiency } \%^{a}\end{array}$ & $\mathrm{k}\left(10^{-4} \min ^{-1}\right)$ & $\begin{array}{l}\text { Half-time } \\
t_{1 / 2}(\min )\end{array}$ \\
\hline- & 0 & 0 & - \\
\hline $\mathrm{SiO}_{2} \mathrm{NP}$ & 0 & 0 & - \\
\hline $\mathrm{NH}_{2}-\mathrm{SiO}_{2} \mathrm{NP}$ & 6.9 & $6.0 \pm 1.3$ & $1150 \pm 250$ \\
\hline $\mathrm{Ag}-\mathrm{SiO}_{2} \mathrm{NP}$ & 13.8 & $11.8 \pm 1.9$ & $590 \pm 95$ \\
\hline $\begin{array}{l}\text { 9ACA conce } \\
1.0 \mathrm{mg} \mathrm{mL}^{-1}\end{array}$ & on $1.0 \times 10^{-5}$ & M; catalyst & oncentration \\
\hline
\end{tabular}

The time evolution of DPBF concentration in the presence of $\mathrm{Ag}-\mathrm{SiO}_{2} \mathrm{NPs}$ under irradiation is spectrophotometrically monitored. Control experiments are performed as well, to ensure the absence of side-reactions.

Negligible bleaching of DPBF absorption is detected when DPBF solution is kept in dark both in the absence and in presence of $\mathrm{Ag}-\mathrm{SiO}_{2} \mathrm{NPs}$ (Fig. S9), thus proving that the reaction is triggered by light. Moreover, the direct irradiation of a DPBF solution without $\mathrm{Ag}-\mathrm{SiO}_{2} \mathrm{NPs}$ for $1 \mathrm{~h}$ demonstrates the substantial photostability of DPBF in the considered time-range (Fig. S10).

Instead, the exposure of DPBF to the light in presence of $\mathrm{Ag}-\mathrm{SiO}_{2} \mathrm{NPs}$ shows considerable bleaching of the scavenger absorption (ca. $50 \%$ in $1 \mathrm{~h}$, see Fig. 4); the kinetic analysis of the DPBF bleaching results in a constant rate of $11.2 \times 10^{-3} \mathrm{~min}^{-1}$. The quantum efficiency of ROS production $\left(\Phi_{\mathrm{ROS}}\right)$ by $\mathrm{Ag}-\mathrm{SiO}_{2} \mathrm{NPs}$ is estimated analysing the DPBF oxidation data, following the procedure described in literature (see also the Supporting information) and using Rose Bengal as a standard $\left(\Phi_{\mathrm{ROS}}=0.76\right.$ [53], Fig. S11). Thus, a value of $17 \%$ has been determined for $\Phi_{\mathrm{ROS}}$ by Ag$\mathrm{SiO}_{2}$ NPs. The reported observations and this value indicate that white light can activate $\mathrm{Ag}-\mathrm{SiO}_{2} \mathrm{NPs}$ and photoinduce the $\mathrm{O}_{2}{ }^{\bullet-}$ and ${ }^{1} \mathrm{O}_{2}$ formation, likely through a charge transfer process between the nanocomposite and the species adsorbed on the silica, as already reported for other materials [54].

In a photocatalytic process in water media, the main ROS species generated are likely $\mathrm{OH}^{\bullet}$ and $\mathrm{O}_{2}{ }^{\bullet-}$. The redox potentials $\left(\mathrm{E}_{\mathrm{H}}\right.$, respect to the normal hydrogen electrode) for their redox couple, $\mathrm{H}_{2} \mathrm{O} / \mathrm{OH}^{\bullet}, \mathrm{O}_{2} / \mathrm{O}_{2}{ }^{--}$, are $2.2 \mathrm{~V}$ and $-0.2 \mathrm{~V}$, respectively. In general, a photocatalyst is able to generate these ROS species $[55,56]$ when: (i) the valence band is at lower energy than $\mathrm{E}_{\mathrm{H}}$ of $\mathrm{OH}^{\bullet}$, thus the photoexcited holes can oxidize $\mathrm{H}_{2} \mathrm{O}$, (ii) the conduction band presents a higher energy value than the $\mathrm{E}_{\mathrm{H}}$ of $\mathrm{O}_{2} / \mathrm{O}_{2}{ }^{--}$, thus the photoexcited electrons own enough reductive power to reduce $\mathrm{O}_{2}$.

Although the irradiation conditions we have used cannot afford the transition from the valence to the conduction band of the silica materials $(9 \mathrm{eV})$ [55], the data indicate that the necessary charge separated species are formed through the activation of silica defects by UV light, as reported elsewhere [57].

Thus, it is likely that defect sites involve intermediate energy states (ca. $4 \mathrm{eV}$ ), located between the valence and conduction band of silica and it is possible to hypothesize that the energies of these charge separated levels are able to satisfy the conditions discussed above in points (i) and / or (ii).

Previously reported experimental evidences demonstrated that, through the excitation of Ag NP plasmons, the direct sensitization of $\mathrm{OH}^{\bullet}$ and $\mathrm{O}_{2}{ }^{\bullet-}$ occurs [56]. However, since DPBF is a selective ROS scavenger, the $\mathrm{Ag}-\mathrm{SiO} 2 \mathrm{NPs}$ most 

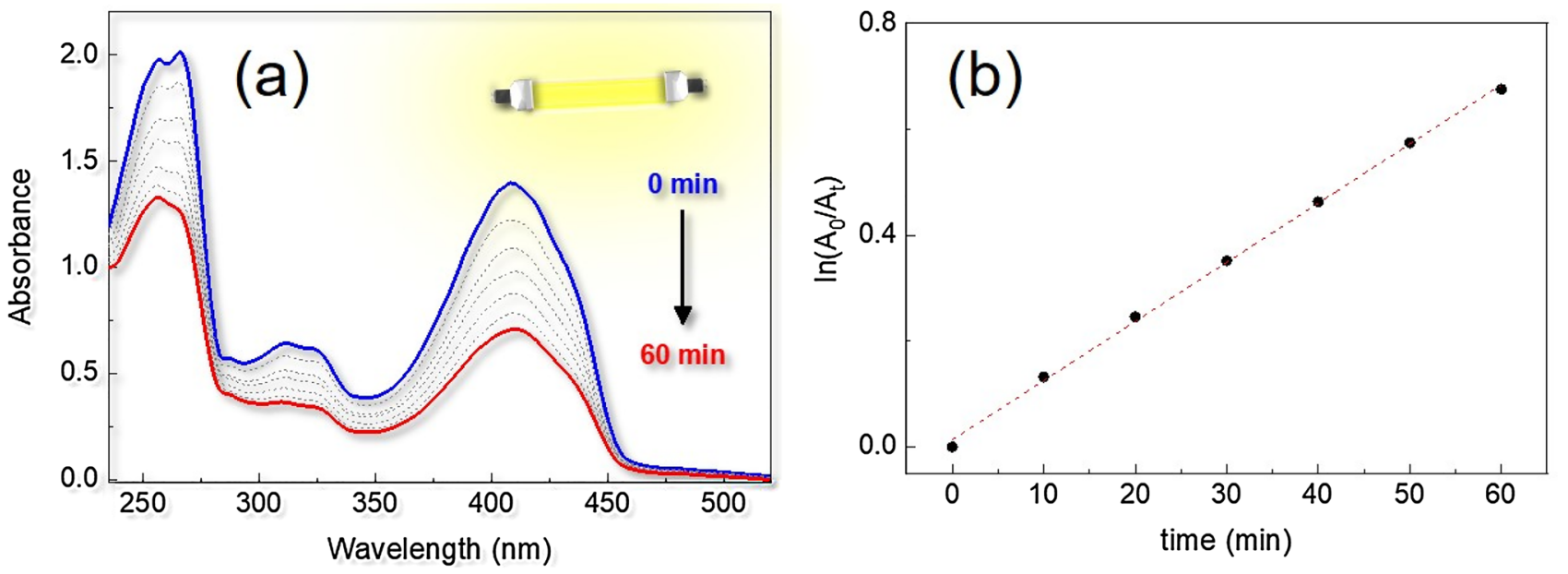

Fig. 4 a DPBF absorption spectra and $\mathbf{b}$ decays at $410 \mathrm{~nm}$ upon irradiation of $\mathrm{Ag}-\mathrm{SiO}_{2}$ NPs with WL. The dotted line in panel b represents the linear fit of the data analyzed through the Equation reported in SI

probably produces $\mathrm{O}_{2}^{\bullet-}$ radicals [52]. Moreover, as discussed in the photodegradation section, the hot carrier generated by the SPR excitation can activate the silica defects, through electron transfer processes, thus creating a situation similar to that under UV irradiation, but with a less energetic irradiation source.

Therefore, using irradiation sources with both UV and visible components, such as solar or artificial light illumination, the photocatalytic efficiency can be enhanced by the combined sensitizing activity of the silver-silica nanocomposite. The capability of $\mathrm{Ag}-\mathrm{SiO}_{2} \mathrm{NPs}$ to form highly oxidative species upon exposure to UV-visible irradiation makes the nanocomposite an excellent candidate for environmental remediation based on controlled photocatalysis.

\section{Conclusion}

We demonstrate the successful application of silver-silica nanocomposites for water photocatalytic remediation.

Mesoporous silica nanoparticles $\left(\mathrm{SiO}_{2} \mathrm{NPs}\right)$ are successfully synthesized with a mean diameter of $120 \mathrm{~nm}$ and a regular channel-like meso-structure, and subsequently functionalized with $\mathrm{NH}_{2}$ groups $\left(\mathrm{NH}_{2}-\mathrm{SiO}_{2} \mathrm{NPs}\right)$. Silver nanoparticles of $10 \mathrm{~nm}$ diameter are anchored on the surface of amino-grafted silica nanoparticles $\left(\mathrm{Ag}-\mathrm{SiO}_{2} \mathrm{NPs}\right)$. The three nanocomposites show electronic transitions in the UV region, due to surface defects of silica, whereas for $\mathrm{Ag}-\mathrm{SiO}_{2}$ NPs the broadening of the extinction spectrum in the visible region is due to the surface plasmon resonance of the metal nanostructures.

A different set of experiments are carried out to determine the photodegradation efficiency of the prepared materials, using 9-anthracenecarboxylic acid (9ACA) as a model compound of aromatic pollutants.

Upon irradiation at $313 \mathrm{~nm}, \mathrm{SiO}_{2} \mathrm{NPs}$ do not show any detectable degradation of 9ACA; $\mathrm{NH}_{2}-\mathrm{SiO}_{2} \mathrm{NPs}$ induced a degradation up to $30 \%$ of anthracene derivative and the effect is even improved when $\mathrm{Ag}-\mathrm{SiO}_{2} \mathrm{NPs}$ are used as catalysts (66\%). Interestingly, also exposure to the $405 \mathrm{~nm}$ radiation successfully activates $\mathrm{Ag}-\mathrm{SiO}_{2} \mathrm{NPs}$ photocatalyst, providing photodegradation of $60 \%$.

The use of different materials and irradiation sources is functional to discern the photocatalytic role of the different components of the nanocomposites and to obtain insight on the photocatalysis mechanism.

The amino groups on the silica surface are fundamental for the photodegradation of 9ACA, since the electrostatic interactions help the negatively charged aromatic molecules to be closer to the positively charged silica surface, a crucial condition for an efficient photocatalysis. It is worth to underline that the presence of Ag NPs enhances the photocatalytic efficiencies even under visible radiation, and very interestingly $\mathrm{Ag}-\mathrm{SiO}_{2} \mathrm{NPs}$ can be activated also by common white fluorescence lamp.

To achieve insight into the catalytic mechanism, the quantum efficiency to generate reactive oxygen species (ROS) is determined. The irradiation of $\mathrm{Ag}-\mathrm{SiO}_{2} \mathrm{NPs}$ suspensions leads to a significant production of ROS with a quantum efficiency of $17 \%$, thus suggesting that the photo-induced degradation of 9ACA is promoted by the photosensitized ROS.

Therefore, silica-based nanomaterials are promising candidates for wastewater remediation, thanks to the synergism between silica and metal nanoparticles that makes the degradation of aromatic pollutants possible under white or solar irradiation. 
Supplementary Information The online version contains supplementary material available at https://doi.org/10.1007/s43630-021-00089-9.

Acknowledgements For this work, no specific funding was received. This work was supported by the Università di Perugia and the Ministero dell'Università e della Ricerca through the program "Dipartimenti di Eccellenza 2018-2022" (grant AMIS). L.L. acknowledges CSGI (Consorzio Interuniversitario per lo Sviluppo dei Sistemi a Grande Interfase, Research Center for Colloid and Surface Science) for the support.

Funding Open access funding provided by Università degli Studi di Perugia within the CRUI-CARE Agreement.

\section{Declarations}

Conflict of interest The authors declare that they have no known competing financial interests or personal relationships that could have appeared to influence the work reported in this paper.

Open Access This article is licensed under a Creative Commons Attribution 4.0 International License, which permits use, sharing, adaptation, distribution and reproduction in any medium or format, as long as you give appropriate credit to the original author(s) and the source, provide a link to the Creative Commons licence, and indicate if changes were made. The images or other third party material in this article are included in the article's Creative Commons licence, unless indicated otherwise in a credit line to the material. If material is not included in the article's Creative Commons licence and your intended use is not permitted by statutory regulation or exceeds the permitted use, you will need to obtain permission directly from the copyright holder. To view a copy of this licence, visit http://creativecommons.org/licenses/by/4.0/.

\section{References}

1. Crini, G., \& Lichtfouse, E. (2019). Advantages and disadvantages of techniques used for wastewater treatment. Environmental Chemistry Letters, 17, 145-155.

2. Gao, Z., et al. (2020). Simultaneous evaporation and decontamination of water on a novel membrane under simulated solar light irradiation. Applied Catalysis B Environmental, 267, 118695.

3. Jiang, M., Qi, Y., Liu, H., \& Chen, Y. (2018). The Role of Nanomaterials and Nanotechnologies in Wastewater Treatment: A Bibliometric Analysis. Nanoscale Research Letters, 13, 233-245.

4. Tarpani, L., et al. (2011). Solid-phase analysis of polycyclic aromatic hydrocarbons by fluorimetric methods. Applied Spectroscopy, 65(12), 1342-1347.

5. Zhang, X., et al. (2019). The fate and enhanced removal of polycyclic aromatic hydrocarbons in wastewater and sludge treatment system: A review. Critical Reviews in Environmental Science and Technology, 49(16), 1425-1475.

6. Fabbri, D., López-Muñoz, M. J. A. D., Medana, C., \& Calza, P. (2019). Photocatalytic abatement of emerging pollutants in pure water and wastewater effluent by $\mathrm{TiO} 2$ and $\mathrm{Ce}-\mathrm{ZnO}$ : Degradation kinetics and assessment of transformation products. Photochemical \& Photobiological Sciences, 18(4), 845-852.

7. Rodriguez-Narvaez, O. M., Peralta-Hernandez, J. M., Goonetilleke, A., \& Bandala, E. R. (2017). Treatment technologies for emerging contaminants in water: A review. Chemical Engineering Journal, 323, 361-380. Elsevier B.V.

8. Zhao, L., et al. (2018). Nanomaterials for treating emerging contaminants in water by adsorption and photocatalysis: Systematic review and bibliometric analysis. Science of the Total Environment, 627, 1253-1263.

9. Belver, C., Bedia, J., Gómez-Avilés, A., Peñas-Garzón, M., \& Rodriguez, J. J. (2019). Semiconductor photocatalysis for water purification. In Nanoscale Materials in Water Purification (pp. 581-651). Elsevier.

10. Zhu, D., \& Zhou, Q. (2019). Action and mechanism of semiconductor photocatalysis on degradation of organic pollutants in water treatment: A review. Environmental Nanotechnology, Monitoring and Management, 12, 100255. Elsevier B.V.

11. Lee, C. Y., Zou, J., Bullock, J., \& Wallace, G. G. (2019). Emerging approach in semiconductor photocatalysis: Towards 3D architectures for efficient solar fuels generation in semi-artificial photosynthetic systems. Journal of Photochemistry and Photobiology C: Photochemistry Reviews, 39, 142-160.

12. Fan, W., \& Leung, M. K. H. (2016). Recent development of plasmonic resonance-based photocatalysis and photovoltaics for solar utilization. Molecules, 21, 180-205.

13. Zhou, Z., et al. (2020). Nitrogen vacancy mediated exciton dissociation in carbon nitride nanosheets: Enhanced hydroxyl radicals generation for efficient photocatalytic degradation of organic pollutants. Journal of Hazardous Materials, 387, 122023.

14. Mano, G., Harinee, S., Sridhar, S., Ashok, M., \& Viswanathan, A. (2020). Microwave assisted synthesis of $\mathrm{ZnO}-\mathrm{PbS}$ heterojuction for degradation of organic pollutants under visible light. Science and Reports, 10(1), 1-14.

15. Singh, P., Abdullah, M. M., \& Ikram S. (2016). Role of nanomaterials and their applications as photo-catalyst andsenors : A review. Nano Reserach and Applications, 2, 1.

16. Liang, Z., Yan, C. F., Rtimi, S., \& Bandara, J. (2019). Piezoelectric materials for catalytic/photocatalytic removal of pollutants: Recent advances and outlook. Applied Catalysis B: Environmental, 241, 256-269.

17. Aggelopoulos, C. A., Dimitropoulos, M., Govatsi, A., Sygellou, L., Tsakiroglou, C. D., \& Yannopoulos, S. N. (2017). Influence of the surface-to-bulk defects ratio of $\mathrm{ZnO}$ and $\mathrm{TiO} 2$ on their UV-mediated photocatalytic activity. Applied Catalysis B: Environmental, 205, 292-301.

18. Jimenez-Relinque, E., \& Castellote, M. (2018). Hydroxyl radical and free and shallowly trapped electron generation and electron/ hole recombination rates in $\mathrm{TiO} 2$ photocatalysis using different combinations of anatase and rutile. Applied Catalysis, A: General, 565, 20-25.

19. Serrà, A., et al. (2019). Highly active $\mathrm{ZnO}$-based biomimetic fern-like microleaves for photocatalytic water decontamination using sunlight. Applied Catalysis B: Environmental, 248, 129-146.

20. Tarpani, L., Bellezza, F., Sassi, P., Gambucci, M., Cipiciani, A., \& Latterini, L. (2019). New insights into the effects of surface functionalization on the peroxidase activity of cytochrome $c$ adsorbed on silica nanoparticles. The Journal of Physical Chemistry B, 123(11), 2567-2575.

21. Fortuni, B., Inose, T., Ricci, M., Fujita, Y., Van Zundert, I., Masuhara, A., Fron, E., Mizuno, H., Latterini, L., Rocha, S., \& Uji-i, H. (2019). Polymeric engineering of nanoparticles for highly efficient multifunctional drug delivery systems. Science and Reports, 9, 2666-2678

22. Latterini, L., \& Tarpani, L. (2011). Hierarchical assembly of nanostructures to decouple fluorescence and photothermal effect. Journal of Physical Chemistry C, 115(43), 21098-21104.

23. Chen, L., Zhang, Y., Chen, G., \& Franco, I. (2018). Stark control of electrons along nanojunctions. Nature Communications, 9(1), 2070.

24. Skuja, L. (1998). Optically active oxygen-deficiency-related centers in amorphous silicon dioxide. Journal of Non-Crystalline Solids, 239, 16-48. 
25. Jafarzadeh, M., Rahman, I. A., \& Sipaut, C. S. (2010). Optical properties of amorphous organo-modified silica nanoparticles produced via co-condensation method. Ceramics International, 36(1), 333-338.

26. Tarpani, L., Ruhlandt, D., Latterini, L., Haehnel, D., Gregor, I., Enderlein, J., \& Chizhik, A. I. (2016). Photoactivation of luminescent centers in single $\mathrm{SiO} 2$ nanoparticles. Nano Letters, 16 , $4312-4316$.

27. Zhou, W., \& Fu, H. (2018). Defect-mediated electron-hole separation in semiconductor photocatalysis. Inorganic Chemistry Frontiers, 5, 1240-1254.

28. Fernández, P., Blanco, J., Sichel, C., \& Malato, S. (2005). Water disinfection by solar photocatalysis using compound parabolic collectors. Catalysis Today, 101, 345-352.

29. Cole, J. R., \& Halas, N. J. (2006). Optimized plasmonic nanoparticle distributions for solar spectrum harvesting. Applied Physics Letters, 89, 153120.

30. Jiang, R., Li, B., Fang, C., \& Wang, J. (2014). Metal / semiconductor hybrid nanostructures for plasmon- enhanced applications. Advanced Materials, 26, 5274-5309.

31. Fu, Y., Li, J., \& Li, J. (2019). Metal/Semiconductor nanocomposites for photocatalysis: fundamentals, structures, applications and properties. Nanomaterials, 9, 359-383.

32. Peiris, S., McMurtrie, J., \& Zhu, H. Y. (2016). Metal nanoparticle photocatalysts: emerging processes for green organic synthesis. Catalysis Science \& Technology, 6(2), 320-338.

33. He, S., Huang, J., Goodsell, J. L., Angerhofer, A., \& Wei, W. D. (2019). Plasmonic Nickel-TiO2 heterostructures for visiblelight-driven photochemical reactions. Angewandte Chemie International Edition, 58(18), 6038-6041.

34. Saravanan, R., et al. (2018). Mechanothermal synthesis of Ag/ $\mathrm{TiO} 2$ for photocatalytic methyl orange degradation and hydrogen production. Process Safety and Environment Protection, 120, 339-347.

35. Raji, R., Sibi, K. S., \& Gopchandran, K. G. (2018). ZnO: Ag nanorods as efficient photocatalysts: Sunlight driven photocatalytic degradation of sulforhodamine B. Applied Surface Science, 427, 863-875.

36. Nosaka, Y., \& Nosaka, A. Y. (2017). Generation and detection of reactive oxygen species in photocatalysis. Chemical Reviews, 117, 11302-11336.

37. Verma, P., Kuwahara, Y., Mori, K., \& Yamashita, H. (2019). Plasmonic catalysis of $\mathrm{Ag}$ nanoparticles deposited on $\mathrm{CeO} 2$ modified mesoporous silica for the nitrostyrene reduction under light irradiation conditions. Catalysis Today, 324, 83-89.

38. Nain, R., Dobhal, S., Bidaliya, P., Saini, G., Pani, B., \& Sirohi, S. (2018). Ag decorated silica nanostructures for surface plasmon enhanced photocatalysis. RSC Advances, 8, 20287-20294.

39. Hanske, C., Sanz-Ortiz, M. N., \& Liz-Marzán, L. M. (2018). Silica-Coated Plasmonic Metal Nanoparticles in Action. Advanced Materials, 30, 1707003.

40. Badr, Y., Abd El-Wahed, M. G., \& Mahmoud, M. A. (2008). Photocatalytic degradation of methyl red dye by silica nanoparticles. Journal of Hazardous Materials, 154(1-3), 245-253.

41. Jadhav, S. A., et al. (2019). Recent advancements in silica nanoparticles based technologies for removal of dyes from water. Colloid Interface Sciences Communications, 30, 100181.
42. Zhang, K., et al. (2013). Facile large-scale synthesis of monodisperse mesoporous silica nanospheres with tunable pore structure. Journal of the American Chemical Society, 135(7), 2427-2430.

43. Zampini, G., Tarpani, L., Massaro, G., Gambucci, M., Nicoziani, A., \& Latterini, L. (2017). Effects of gold colloids on the photosensitization efficiency of silica particles doped with protoporphyrin IX. ChemPhotoChem, 1(12), 553-561.

44. Abdel-Mottaleb, M. S. A., et al. (2000). Fluorescence and photostability studies of anthracene-9-carboxylic acid in different media. International Journal of Photoenergy, 2, 47-53.

45. Selvaggi, R., Tarpani, L., Santuari, A., Giovagnoli, S., \& Latterini, L. (2015). Silica nanoparticles assisted photodegradation of acridine orange in aqueous suspensions. Applied Catalysis B: Environmental, 168, 363-369.

46. Xu, S., et al. (2015). Tuning the singlet-triplet energy gap: A unique approach to efficient photosensitizers with aggregationinduced emission (AIE) characteristics. Chemical Science, 6(10), 5824-5830.

47. Zampini, G., et al. (2019). Experimental evidences on the role of silica nanoparticles surface morphology on the loading, release and activity of three proteins. Microporous and Mesoporous Materials, 287, 220-227.

48. Zampini, G., et al. (2017). Morphology effects on singlet oxygen production and bacterial photoinactivation efficiency by different silica-protoporphyrin IX nanocomposites. RSC Advances, 7(24), 14422-14429.

49. Spallino, L., et al. (2014). Visible-ultraviolet vibronic emission of silica nanoparticles. Physical Chemistry Chemical Physics: PCCP, 16(40), 22028-22034.

50. Skuja, L. (1992). Time-resolved low temperature luminescence of non-bridging oxygen hole centers in silica glass. Solid State Communications, 84(6), 613-616.

51. Sarina, S., Waclawik, E. R., \& Zhu, H. (2013). Photocatalysis on supported gold and silver nanoparticles under ultraviolet and visible light irradiation. Green Chemistry, 15(7), 1814-1833. Royal Society of Chemistry.

52. Burns, J. M., et al. (2012). Methods for reactive oxygen species (ROS) detection in aqueous environments. Aquatic Sciences, 74, 683-734.

53. Redmond, R. W., \& Gamlin, J. N. (1999). A compilation of singlet oxygen yields from biologically relevant molecules. Photochemistry and Photobiology, 70(4), 391-475.

54. Zhang, J., \& Nosaka, Y. (2014). Mechanism of the OH radical generation in photocatalysis with $\mathrm{TiO} 2$ of different crystalline types. Journal of Physical Chemistry C, 118, 10824-10832.

55. Li, Y., Zhang, W., Niu, J., \& Chen, Y. (2012). Mechanism of photogenerated reactive oxygen species and correlation with the antibacterial properties of engineered metal-oxide nanoparticles. ACS Nano, 6(6), 5164-5173.

56. Zhang, W., Li, Y., Niu, J., \& Chen, Y. (2013). Photogeneration of reactive oxygen species on uncoated silver, gold, nickel, and silicon nanoparticles and their antibacterial effects. Langmuir, 29(15), 4647-4651.

57. Munekuni, S., et al. (1990). Various types of nonbridging oxygen hole center in high-purity silica glass. Journal of Applied Physics, 68(3), 1212-1217. 


\section{Authors and Affiliations}

\section{G. Romolini ${ }^{1,2} \cdot$ M. Gambucci $^{1} \cdot$ D. Ricciarelli ${ }^{1} \cdot$ L. Tarpani $^{1} \cdot$ G. Zampini $^{1} \cdot$ L. Latterini $^{1}$ (D)}

G. Zampini

giulia.zampini87@gmail.com

$\triangle$ L. Latterini loredana.latterini@unipg.it

1 Department of Chemistry, Biology and Biotechnology, University of Perugia, Via Elce di Sotto 8, 06123 Perugia, Italy
2 Present Address: Chem \& Tech, Molecular Imaging and Photonics, KULeuven, Celestijnenlaan $200 \mathrm{~F}$, B-3001 Leuven, Belgium 